\title{
VARIAÇÕES DO CICLO ANUAL DA TEMPERATURA DA SUPERFÍCIE DO MAR NO PACÍFICO TROPICAL
}

\author{
RENATO ORRÚ PEDROSO GERÓLAMO E MARY TOSHIE KAYANO
}

\author{
Instituto Nacional de Pesquisas Espaciais (INPE) São José dos Campos, SP, Brasil \\ renato.orru@cptec.inpe.br, mary.kayano@cptec.inpe.br
}

Recebido Setembro 2008 - Aceito Outubro 2009

\begin{abstract}
RESUMO
Variações do ciclo anual da Temperatura da Superfície do Mar (TSM) do Pacífico Tropical entre $20^{\circ} \mathrm{N}$ e $20^{\circ} \mathrm{S}$, para o período de 1854 a 2006, foram estudadas usando análises de ondaletas. O estudo mostrou que o ciclo anual, considerando-se a variância para em torno de 1 ano, de TSM possui variabilidade interanual e interdecenal. Existem indicações de que a maior parte das variações interanuais no ciclo anual de TSM das áreas do hemisfério Norte (HN) e do hemisfério Sul (HS), está relacionada ao ciclo do El Niño-Oscilação Sul (ENOS). A maioria dos picos da Média por Escalas da Potência localizada da ondaleta (MEPO) para a escala entre 0,7 e 1,2 anos, para as áreas de ambos os hemisférios, coincide com extremos do ENOS. Os Espectros da Potência Global (EPGs) mostraram variância máxima do ciclo anual de TSM entre $120^{\circ} \mathrm{E}$ e $140^{\circ} \mathrm{E}$ na faixa do $\mathrm{HN}$ (entre equador e $20^{\circ} \mathrm{N}$ ), e variância crescente da linha da data para leste com o máximo entre $100^{\circ} \mathrm{W}$ e $80^{\circ} \mathrm{W}$ na faixa do HS (entre equador e $20^{\circ} \mathrm{S}$ ). Portanto, o ciclo anual de TSM apresenta variações entre os HN e HS com a máxima variância localizando-se no hemisfério leste para o HN e no hemisfério oeste para o HS.

Palavras-chave: Ciclo anual de TSM, Pacífico Tropical, Ondaletas.
\end{abstract}

\begin{abstract}
ANNUAL CYCLE VARIATIONS OF SEA SURFACE TEMPERATURE IN THE TROPICAL PACIFIC

Annual cycle variations of the sea surface temperature (SST) in the Tropical Pacific Ocean between $20^{\circ} \mathrm{N}$ and $20^{\circ} \mathrm{S}$ for the period from 1854 to 2006 were studied using wavelet analysis. The study showed that the SST annual cycle, considered the variance around 1 year, contains interannual and interdecadal variabilities. There are indications that most of the SST annual cycle variations at Northern Hemisphere ( $\mathrm{NH}$ ) and at Southern Hemisphere (SH) areas is related to the El Niño-Southern Oscillation (ENSO) cycle. Most peaks of the localized Scale Average Power (SAP) for the 0.7-1.2 year scale for the areas on both hemispheres coincides with ENSO extremes. The Wavelet Power Spectra (WPS) shows maximum variance of the SST annual cycle between $120^{\circ} \mathrm{E}$ and $140^{\circ} \mathrm{E}$ for the northern hemisphere $(\mathrm{NH})$ band (between equator and $20^{\circ} \mathrm{N}$ ), and eastward increasing variance from the dateline with a maximum between $100^{\circ} \mathrm{W}$ and $80^{\circ} \mathrm{W}$ for the southern hemisphere (SH) band (between equator and $20^{\circ} \mathrm{S}$ ). Therefore, the SST annual cycle presents variations between the $\mathrm{NH}$ and $\mathrm{SH}$ with the maximum variance being located in the eastern hemisphere for the $\mathrm{NH}$ and in the western hemisphere for the SH.
\end{abstract}

Keywords: Annual Cycle of SST, Tropical Pacific, Wavelets. 


\section{INTRODUÇÃO}

O oceano tem um papel importante na modulação da variabilidade climática através de processos dinâmicos e termodinâmicos envolvidos na complexa interação entre este e a atmosfera. Isso se deve ao fato de o oceano servir como uma forçante para a atmosfera, bem como por ocupar um terço da superfície terrestre e também por controlar as trocas de calor latente e sensível (Kayano et al., 2005). Assim a Temperatura da Superfície do Mar (TSM) nos oceanos tem um papel preponderante na variabilidade climática interanual a interdecenal, por ser uma das principais variáveis físicas envolvidas na interação oceano-atmosfera. Na escala interanual, o padrão de variabilidade desta variável no Pacífico leste equatorial caracteriza-se nos eventos quentes (frios), denominados de El Niño (La Niña), pela presença de anomalias positivas (negativas). Os padrões anômalos da circulação atmosférica associados a estes padrões de anomalias de TSM, hoje conhecidos como padrões que fazem parte dos ciclos do fenômeno El Niño-Oscilação Sul (ENOS), conduzem a padrões climáticos anômalos em várias áreas do globo que se manifestam em diversas variáveis como a precipitação, temperatura do ar, pressão ao nível médio do mar entre outras (Bjerknes, 1969; Rasmusson e Carpenter, 1982; Ropelewski e Halpert, 1987 e 1996). No caso do Brasil, diversos autores documentaram duas áreas nas quais as anomalias de precipitação são coerentes com o ciclo do ENOS: o nordeste cujas secas (chuvas excessivas) e o sul cujas chuvas excessivas (secas) estão associadas à ocorrência de El Niño (La Niña) (Ropelewski e Halpert, 1987, 1989; Aceituno, 1988; Kayano et al., 1988; Kousky e Ropelewski, 1989; Rao e Hada, 1990; Grimm et al. 1998; Grimm et al., 2000, entre outros).

Nos estudos da variabilidade do clima, um procedimento comum consiste em se remover das variáveis utilizadas a variância associada ao ciclo anual, definido pelas médias mensais de longo prazo, embora uma parte substancial da variância total encontra-se nessa escala de tempo. Isso decorre do fato da análise da variabilidade ser seriamente dificultada pela presença do ciclo anual. A remoção do ciclo anual permite que outras flutuações presentes na série temporal se tornem mais evidentes e estatisticamente significativas. Porém, o ciclo anual de uma variável pode apresentar variações dependendo do período considerado como base para seu cálculo. Isso ocorre principalmente se a série temporal contiver flutuações de baixa freqüência, como por exemplo, flutuações multidecenais. Os valores das normais climatológicas podem ser distintos, se o período base do cálculo do ciclo anual se estender grandemente para uma ou outra fase da flutuação multidecenal, ou mesmo incluir ambas as fases desta oscilação. Exemplos de variações interdecenais das normais climatológicas de 30 anos de TSM, em diversas áreas do Pacífico, podem ser encontrados em Xue et al. (2003). Uma conseqüência óbvia da variabilidade do ciclo anual de uma variável, é que os valores de suas anomalias, e mesmo seu sinal, podem ser alterados dependendo do período considerado para o cálculo do ciclo anual. Isso aponta para a importância em se estudar a variabilidade do ciclo anual de TSM, em particular do Pacífico tropical, pois é essa a região primária da manifestação do ENOS, principal fenômeno envolvido na variabilidade interanual do clima.

Neste contexto, estudos recentes apontaram que o ciclo anual da TSM do Pacífico equatorial pode apresentar variações em escalas interanual (Gu e Philander, 1995; Setoh et al., 1999; Kim e Chung, 2001) e interdecenal (Setoh et al., 1999; Xue et al., 2003). Gu e Philander (1995), usando análises de ondaletas, mostraram que a amplitude do ciclo anual de TSM do Oceano Pacífico tropical leste varia muito mais em uma escala temporal interanual do que decenal. A variabilidade do ciclo anual de TSM, na escala decenal ou de mais baixa freqüência, pode refletir também as interações entre os modos de variabilidade interanual e anual de TSM do Pacífico. Existem evidências observacionais destas interações embora sua natureza seja pouco conhecida. Um exemplo disto é que os eventos El Niño iniciaram em regiões e meses distintos nos períodos entre 1950 e 1980 e após 1980. De fato, os eventos El Niño que ocorreram entre 1950 e 1980 desenvolveram-se no Pacífico equatorial leste entre março e abril (Rasmusson e Carpenter, 1982), e os que ocorrem após 1980 iniciaram no Pacífico equatorial oeste em torno de setembro-outubro (Wang, 1995). Portanto, durante os desenvolvimentos destes eventos, as anomalias de TSM propagaram-se para leste no primeiro caso, e em direção oposta no segundo caso.

Um outro aspecto é que o fenômeno ENOS e o ciclo anual tendem a estar em fase. Rasmusson e Arkin (1985) observaram que em algumas regiões do Pacífico equatorial, o sinal do El Niño se iguala ou excede a amplitude do ciclo anual médio, de modo que o El Niño representa uma pequena perturbação do ciclo anual médio. Estes autores notaram mesmo nas regiões com o sinal relativamente forte do El Niño que a evolução das anomalias em um tempo de vida de 18 a 24 meses para um episódio típico mostrava uma forte tendência para estar em fase com o ciclo anual. Assim, Rasmusson e Arkin (1985) sugeriram que o aparecimento, evolução e enfraquecimento das anomalias associadas ao ENOS podem ser interpretados em termos de deslocamento de fase ou modulação da amplitude de algumas características do ciclo anual das variáveis envolvidas. Em um estudo mais recente, Wang e Wang (1996) mostraram que tanto o aquecimento, como o resfriamento anômalo das águas superficiais no Pacífico equatorial leste, alcançam seu máximo na estação fria deste setor oceânico, de setembro a janeiro, resultando em ciclo do ENOS em fase com o ciclo do 
anual de TSM do Pacífico tropical. Portanto, estes resultados reforçam que o estudo do ciclo anual de TSM no Pacífico reveste-se de importância para o melhor entendimento das interações entre os modos de variabilidade interanual e anual de TSM, neste setor oceânico.

As relações entre o ciclo anual e a variabilidade de TSM no Pacífico tropical têm sido investigadas nos últimos anos. Por exemplo, Gu e Philander (1995) encontraram que o ENOS influencia o ciclo anual de TSM no Pacífico tropical central e leste. Esta influência é tal que o ciclo anual é fraco em anos de El Niño e forte em anos de La Niña. No entanto, Setoh et al. (1999), usando análises de ondaletas, mostraram que esta relação depende da região e período considerados. Por exemplo, eles não encontraram evidências desta relação nos anos 80 e 90. Setoh et al. (1999) mostraram também, que a amplitude do ciclo anual de TSM no Pacífico equatorial é crescente de oeste para leste com um máximo em $100^{\circ} \mathrm{W}$.

Do acima exposto, torna-se evidente que um melhor entendimento do ciclo anual de TSM no Pacífico tropical, através de análises mais detalhadas do mesmo bem como de sua variabilidade, poderá contribuir para os estudos sobre a variabilidade interanual de TSM deste oceano bem como sobre a interação desta com o ciclo anual correspondente. Análises do ciclo anual de TSM, dividindo a série em períodos de mesmo número de anos, como a apresentada por Xue et al. (2003), pressupõem que o ciclo anual não varia dentro de cada período. No entanto, isso nem sempre é válido. Assim sendo é necessário usar uma técnica que permita levar em conta a variação do ciclo anual. Uma destas técnicas faz uso da transformada de ondaleta (TO), que é uma alternativa à Transformada Fourier para analisar fenômenos locais, não-periódicos e multiescalares. Esta técnica permite analisar escalas diferentes de variabilidade temporal e não necessita de uma série estacionária. Em outras palavras, a TO decompõe uma série temporal em espaço tempo-freqüência, de modo a ser possível determinar os modos dominantes de variabilidade e como estes variam no tempo, mantendo simultaneamente a localização temporal dos principais eventos. Assim, é apropriada para analisar eventos irregularmente distribuídos e séries temporais que contêm potência nãoestacionária em várias freqüências diferentes (Torrence e Compo, 1998). A variabilidade de índices, que descrevem o fenômeno do ENOS, foi analisada usando ondaletas (Wang e Wang, 1996; Torrence e Compo, 1998).

Estudos anteriores sobre a variabilidade do ciclo anual de TSM no Pacífico têm se restringido ao longo do equador ( $\mathrm{Gu}$ e Philander, 1995) ou em uma estreita faixa ao longo desta latitude (Setoh et al., 1999). Assim, diferenças no comportamento do ciclo anual de TSM entre o HN e HS, bem como o ciclo anual em faixas mais largas, que incluam os centros de ação de TSM, como o do Pacífico norte leste ("cold tongue") dominado por águas frias ou o do Pacífico norte oeste dominado por águas quentes (piscina quente), não foram ainda estudados. Assim o presente estudo visa documentar a variabilidade do ciclo anual de TSM no Pacífico tropical (entre $20^{\circ} \mathrm{N}$ e $20^{\circ} \mathrm{S}$ ), bem como sua relação com o ENOS. Em vista das vantagens do uso da TO mencionadas acima, neste estudo esta é a técnica usada. Vale ressaltar, que aqui as análises são restritas ao ciclo anual, uma vez que valores de anomalias são em parte dependentes de valores climatológicos, e também porque análises destas se referem à análise de variabilidade temporal para escalas maiores do que o ciclo anual, uma vez que este é removido da série. Assim, análise da variabilidade das anomalias está fora do escopo do presente trabalho.

\section{DADOS E METODOLOGIA}

Os dados usados neste trabalho consistem de séries mensais de TSM globais obtidas do sítio http://www.jisao. washington.edu/data/ersst/\#data, para o período de 1854 até 2006. Estas séries de TSM foram reconstruídas por Smith e Reynolds (2004) em pontos de grade no globo com uma resolução espacial de $2^{\circ}$ por $2^{\circ}$ em latitude e longitude. A

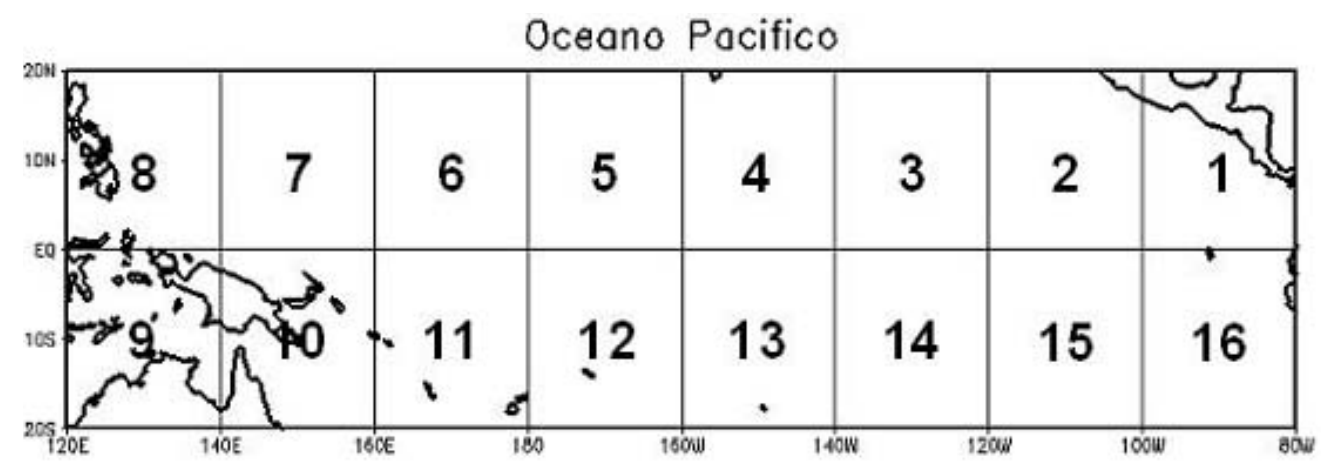

Figura 1 - Área dos 16 índices de TSM. 
região analisada é delimitada entre as latitudes $20^{\circ} \mathrm{S}$ e $20^{\circ} \mathrm{N}$ e longitudes $120^{\circ} \mathrm{E}$ e $80^{\circ} \mathrm{W}$.

São obtidos 16 índices de TSM no Pacífico para todo o período de disponibilidade de dados, calculando-se a média de TSM dos pontos de grade em áreas selecionadas de $20^{\circ}$ em longitude por $20^{\circ}$ em latitude. A primeira destas áreas no $\mathrm{HN}$ é delimitada pelo equador, $20^{\circ} \mathrm{N}, 80^{\circ} \mathrm{W}$ e $100^{\circ} \mathrm{W}$, as áreas subseqüentes no mesmo hemisfério estão a cada $20^{\circ} \mathrm{em}$ longitude, e as áreas do HS simétricas a estas, estão entre o equador e $20^{\circ} \mathrm{S}$, como ilustradas na Figura 1 . Pelo exposto acima, a escolha destas áreas considera as regiões dos centros de ação de TSM, e assim como estas tenham aproximadamente o mesmo número de pontos de grade sobre o oceano. Algumas destas áreas incluem alguns poucos pontos de grade sobre continentes ou ilhas. Ressalta-se que a análise de 8 áreas subseqüentes no HN e 8 áreas também subseqüentes no HS permite avaliar as variações em longitude do ciclo anual e as diferenças entre o $\mathrm{HN}$ e HS para uma mesma faixa de longitude. Para simplificar o texto e não torná-lo muito repetitivo serão apresentadas e discutidas apenas 4 áreas de cada hemisfério.

Como através da TO de uma série é possível obter informações da amplitude de quaisquer sinais dentro da série, bem como de que forma esta amplitude varia com o tempo (Torrence e Compo, 1998), os 16 índices são analisados usando TO. Em virtude das variações de TSM serem suaves, a ondaleta de Morlet é a que melhor descreve tais variações e vem sendo usada para decompor tais séries no espaço tempo-frequência (Gu e Philander, 1995; Torrence e Compo, 1998; Setoh et al.., 1999). Assim a ondaleta de Morlet é a usada no presente trabalho. Uma breve descrição desta ondaleta e dos cálculos envolvidos são fornecidos a seguir.

A ondaleta de Morlet é uma função exponencial complexa modulada por uma Gaussiana, $e^{i \omega_{0} \eta} e^{-\eta^{2} / 2}$, com $\eta=t / s$, onde $t$ é o tempo, $s$ é a escala da ondaleta e $\omega_{0}$ é uma freqüência não dimensional. O procedimento computacional da análise de ondaleta usada aqui é a descrita por Torrence e Compo (1998). Vale mencionar que a função de ondaleta em cada escala $s$ é normalizada por $s^{-1 / 2}$ para se ter energia unitária, para permitir comparações entre escalas, bem como com a transformada de ondaleta de outras séries (Torrence e Compo, 1998).

Por razões computacionais, a série é preenchida com zeros até que seu comprimento atinja a próxima potência de 2. Em conseqüência, há descontinuidades no final da série e os valores do espectro de potência da ondaleta para as escalas grandes não são confiáveis. Assim, Torrence e Compo (1998) definiram o Cone de Influência (COI), região do espectro de potência (curva em forma de U) sob a qual o efeito de borda é importante, como o tempo de decaimento (e-folding time) para a auto-correlação da potência da ondaleta em cada escala. Este tempo é escolhido de modo que a potência da ondaleta para uma descontinuidade no final da série caia por um fator de $e^{-2}$, de modo que além daquele ponto, os efeitos de borda são desprezíveis. Valores do espectro de potência da ondaleta que caírem dentro COI não devem ser analisados (Torrence e Compo, 1998).

Da análise de ondaletas de uma série temporal resultam os espectros de potência (ou variância) local da ondaleta (EPO). Para uma dada escala e tempo, nas análises do presente estudo o nível de confiança da significância estatística do EPO adotado é o de 95\%. Nesta análise, o Espectro de Potência Global (EPG) de cada índice, que é a média em tempo da EPO para uma escala fixa, é similar ao espectro de potência de Fourier (Torrence e Compo, 1998). A variabilidade temporal do ciclo anual de cada índice é estudada através da Média para uma faixa de Escalas da EPO (MEPO). No presente estudo a escala considerada é de

Tabela 1 - Eventos de El Niño e La Niña do período 1854 - 2006. O primeiro ano se refere ao de surgimento do evento e o segundo ao de decaimento.

\begin{tabular}{|c|c|}
\hline $\begin{array}{c}\text { EVENTOS DE } \\
\text { EL NINO }\end{array}$ & $\begin{array}{c}\text { EVENTOS DE } \\
\text { LA NINA }\end{array}$ \\
\hline $1888-1889$ & $1856-1857$ \\
\hline $1899-1900$ & $1873-1874$ \\
\hline $1902-1903$ & $1879-1880$ \\
\hline $1904-1906$ & $1886-1887$ \\
\hline $1911-1912$ & $1889-1890$ \\
\hline $1914-1915$ & $1892-1894$ \\
\hline $1923-1924$ & $1898-1899$ \\
\hline $1925-1926$ & $1903-1904$ \\
\hline $1930-1931$ & $1906-1907$ \\
\hline $1939-1942$ & $1908-1911$ \\
\hline $1951-1952$ & $1915-1918$ \\
\hline $1953-1954$ & $1920-1921$ \\
\hline $1957-1958$ & $1922-1923$ \\
\hline $1963-1964$ & $1924-1925$ \\
\hline $1965-1966$ & $1926-1927$ \\
\hline $1968-1970$ & $1932-1934$ \\
\hline $1972-1973$ & $1938-1939$ \\
\hline $1976-1977$ & $1942-1943$ \\
\hline $1979-1980$ & $1944-1945$ \\
\hline $1982-1983$ & $1948-1951$ \\
\hline $1986-1988$ & $1954-1956$ \\
\hline $1990-1992$ & $1964-1965$ \\
\hline $1993-1995$ & $1970-1972$ \\
\hline $1997-1998$ & $1973-1976$ \\
\hline $2002-2005$ & $1984-1985$ \\
\hline & $1988-1989$ \\
\hline & $1998-2000$ \\
\hline
\end{tabular}


0,7 a 1,2 anos. Assim no texto que se segue, a MEPO é definida como a soma ponderada do EPO da escala $s_{1}=0,7$ a $s_{2}=1,2$. A escala de 0,7 a 1,2 anos foi considerada a escala anual por Gu e Philander (1995), e é usada neste mesmo contexto no presente trabalho. Assim, nos capítulos de resultados e conclusões, as variações da MEPO se referem às variações do ciclo anual. Vale ressaltar que as séries são normalizadas pelos correspondentes desvios padrão antes de se proceder aos cálculos da TO. Assim, os símbolos $\sigma^{2}$ e $\sigma$, daqui em diante, se referem ao desvio padrão e variância, respectivamente.

Para analisar a relação do ciclo anual com o ENOS, os anos de ocorrência de El Niño e de La Niña são identificados no índice de anomalia de TSM para a região do Niño 3.4 suavizado com uma média móvel de 5 meses. De acordo com Trenberth (1997), se este índice exceder $0,4^{\circ} \mathrm{C}$ (for menor do que $-0,4^{\circ} \mathrm{C}$ ) por 6 meses consecutivos ou mais, tem-se a ocorrência de um evento El Niño (La Niña). A Tabela 1 que lista os anos de El Niño e La Niña usa este critério.

\section{RESULTADOS}

As Figuras 2 a 10 mostram as análises de ondaletas para os índices de TSM de áreas selecionadas do Pacífico tropical indicadas nestas figuras e cujas localizações encontram-se na Figura 1. Nestas figuras, nos painéis (a) são apresentados os EPO, nos painéis (b), os EPG, e os gráficos em (c) apresentam a MEPO para a escala de 0,7 a 1,2 anos.
O EPG de todas as áreas do HN mostra um pico dominante na escala de aproximadamente 1 ano, e resulta de EPOs significativos para a escala do ciclo anual que ocorrem quase ininterruptamente de 1854 a 2006, exceto em curtos períodos com valores não significativos de EPO nesta escala. As análises dos gráficos de EPG no $\mathrm{HN}$ para a escala anual, mostram valores em torno de $2 \sigma$ para as áreas 1 e 2 , de $5 \sigma$ para as áreas 3, 4 e 7, de $6 \sigma$ para as áreas 5 e 6, e de 7 $\sigma$ na área 8. É notável que, exceto pela área 7 , a variância do ciclo anual no HN apresenta gradual aumento de leste para oeste em toda a faixa, culminado com a máxima variância entre $120^{\circ} \mathrm{E}$ e $140^{\circ} \mathrm{E}$, na região de piscina de águas quentes do Pacífico tropical norte e oeste. Os EPGs nas áreas 1 (Figura 2), 2 e 8 (Figuras não mostradas) apresentam uma pequena variabilidade semianual (escala de 0,5 anos). As Figuras 2 a 4 mostram picos secundários (não-significativos) para a escala interanual (5 anos), que resultam de EPO relativamente altos dos correspondentes índices, mas não significativos nos períodos de 1890-1920, 1950-2000 para a escala de 3-7 anos. Este fato é notado nas áreas 1 a 5. Vale ressaltar que a variabilidade na escala interanual não é significativa nesta análise em vista de que o ciclo anual é mantido nos índices. O pico interanual gradualmente se reduz para áreas mais a oeste da área 5 (Figuras 4 e 5). Isso significa que a variabilidade interanual diminui a partir da linha da data para oeste no Pacífico tropical norte. Portanto, o sinal do ENOS no Pacífico tropical norte é forte no seu setor central e leste, diminuindo da linha da data para oeste.
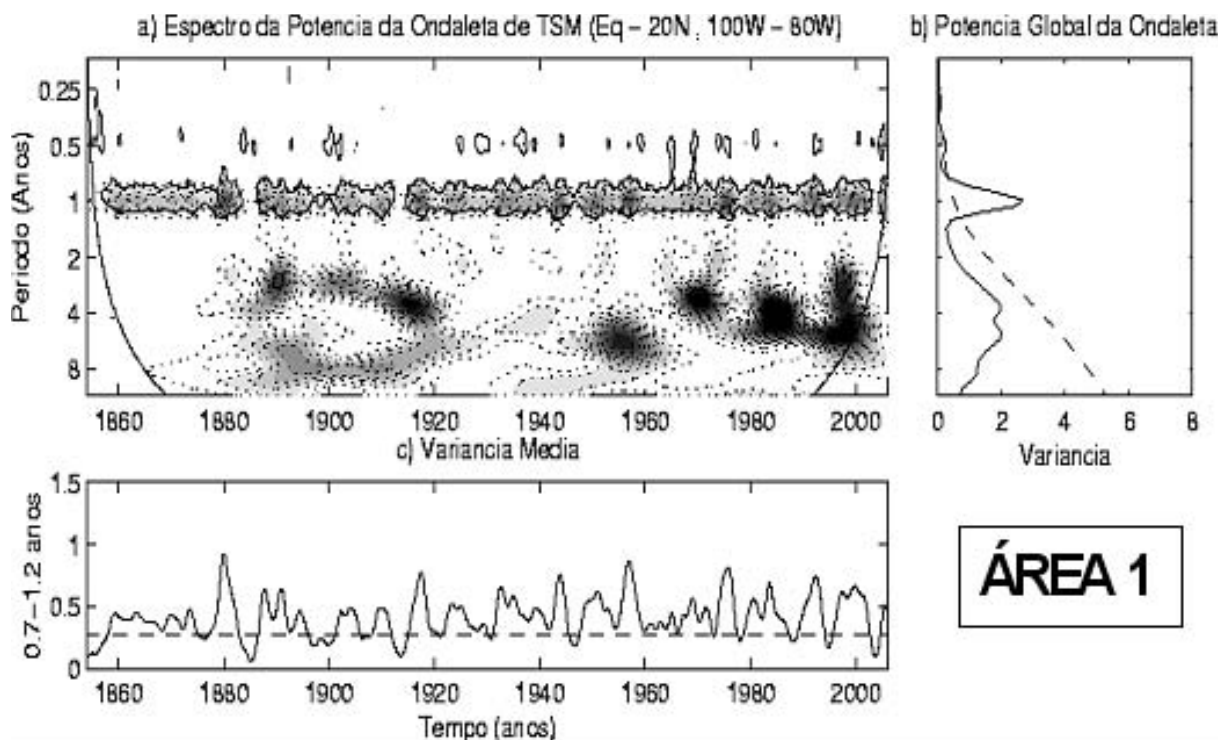

Figura 2 - a) EPO para o índice de TSM da área 1, normalizado por $\sigma^{2}=0,3202$; b) EPG; c) MEPO na banda de 0,7 - 1,2 anos. Em a) as áreas sombreadas representam variâncias normalizadas, variando de 0 a 20 com o intervalo de contorno de 2, a curva em forma de U representa o COI, e os contornos fechados indicam áreas com EPO significativo ao nível de confiança de 95\%. Em (b), à direita da curva tracejada, o EPG é significativo ao nível de confiança de 95\%. Em (c), acima da reta tracejada a MEPO é significativo ao nível de confiança de $95 \%$. 
As MEPOs para a escala de 0,7 - 1,2 anos (painel (c)) e a EPO na escala interanual em todas as áreas do HN, mostram fraca variabilidade anual e praticamente ausência do sinal da variabilidade interanual para o período anterior a 1880. Smith e Reynolds (2004) alertaram que os dados de TSM neste período têm uma fraca variabilidade interanual devido à limitação na reconstrução desses dados, ocasionada pela escassez de dados observados anterior a 1880. A MEPO da área 1 mostra picos nos anos de 1880, 1918, 1942, 1958, 1976, 1993 e 2000, possivelmente associados a uma variabilidade de uma escala de 20-40 anos. A esta variabilidade estão superimpostos picos na MEPO na escala interanual. Portanto, o ciclo anual do índice da área 1 apresenta variabilidade interanual e multidecenal (Figura 2).

Na MEPO da área 2 destacam-se dois picos, um por volta de 1888 e o segundo em 1942, aos quais estão superimpostos picos de menores amplitudes na escala interanual (Figura não mostrada). De forma semelhante, as MEPOs das áreas 3 (Figura 3) e 4 (Figura não mostrada) apresentam vários picos na escala interanual, cujas amplitudes não excedem $1 \sigma$. Portanto, o ciclo anual dos índices das áreas 2, 3 e 4 apresenta variabilidade interanual superimposta a variabilidade de mais baixa freqüência. As MEPOs das áreas 5 (Figura 4), 6 e 7 (Figura 5) apresentam maior variabilidade do ciclo anual do que as das áreas à leste, indicada pela dominância nos gráficos da MEPO de valores excedendo $0,5 \sigma$ bem como por vários picos com amplitudes superiores a $1 \sigma$. Por exemplo, na área 5 há picos em 1911, 1920, 1992 e 2000 com valores superiores a $1 \sigma$, dos quais os de 1911 e 1992 coincidem com anos de ocorrência de El Niño e os de 1920 e 2000 com anos de La Niña (Figura 4 e Tabela 1). Nas áreas 6 (Figura não mostrada) e 7 (Figura 5) ocorrem as maiores variabilidades para todo período, com os picos superiores a $1 \sigma$. De fato, na área 6 é notável um pico de $1,4 \sigma$ em 1911 (Figura não mostrada) e na área 7 ocorrem picos de 1,2 $\sigma$ em 1918, 1969 e 1992 (Figura 5). Destes anos, o primeiro coincide com ano de La Niña e os dois últimos anos com anos de ocorrência de El Niño (Tabela 1). Na área 6 observam-se ainda, picos superiores a $1 \sigma$ em 1907, 1911, 1920, 1931, 1937, 1960, 1985 e 1992 (Figura não mostrada). De forma semelhante, a área 7 também apresenta diversos picos com valores superiores a 16, como em 1908, 1913, 1918, 1930, 1938, 1958, 1969, 1992 e 1996, dos quais os anos de 1930, 1958, 1969 e 1992 coincidem com anos de ocorrência de El Niño e os de 1908, 1918 e 1938 com anos de ocorrência de La Niña (Figura 5 e Tabela 1). Essa variabilidade diminui na área 8, mas os picos em 1969 e 1996 mantêm-se em torno 1,2 $\sigma$ (Figura não mostrada). Portanto, o ciclo anual dos índices do Pacífico tropical norte entre $140^{\circ} \mathrm{E}$ e $160^{\circ} \mathrm{W}$, apresenta forte variabilidade interanual e de mais baixa freqüência do que os índices em áreas mais a leste nesta mesma faixa latitudinal. Em alguns casos, o aumento da variabilidade interanual do ciclo anual parece estar relacionado à ocorrência de El Niño, como mostrado nas áreas 5 e 7 (Figura 4 e 5).

As Figuras 6 a 10 ilustram os gráficos de EPO, EPG e MEPO das áreas 9, 11, 12, 14 e 16, respectivamente. Nestas Figuras e nas demais não mostradas do HS é notável um pico dominante no gráfico de EPG na escala de aproximadamente 1 ano, que resulta de EPO significativo em todo o período de estudo. Os valores de EGP na escala de tempo anual nas
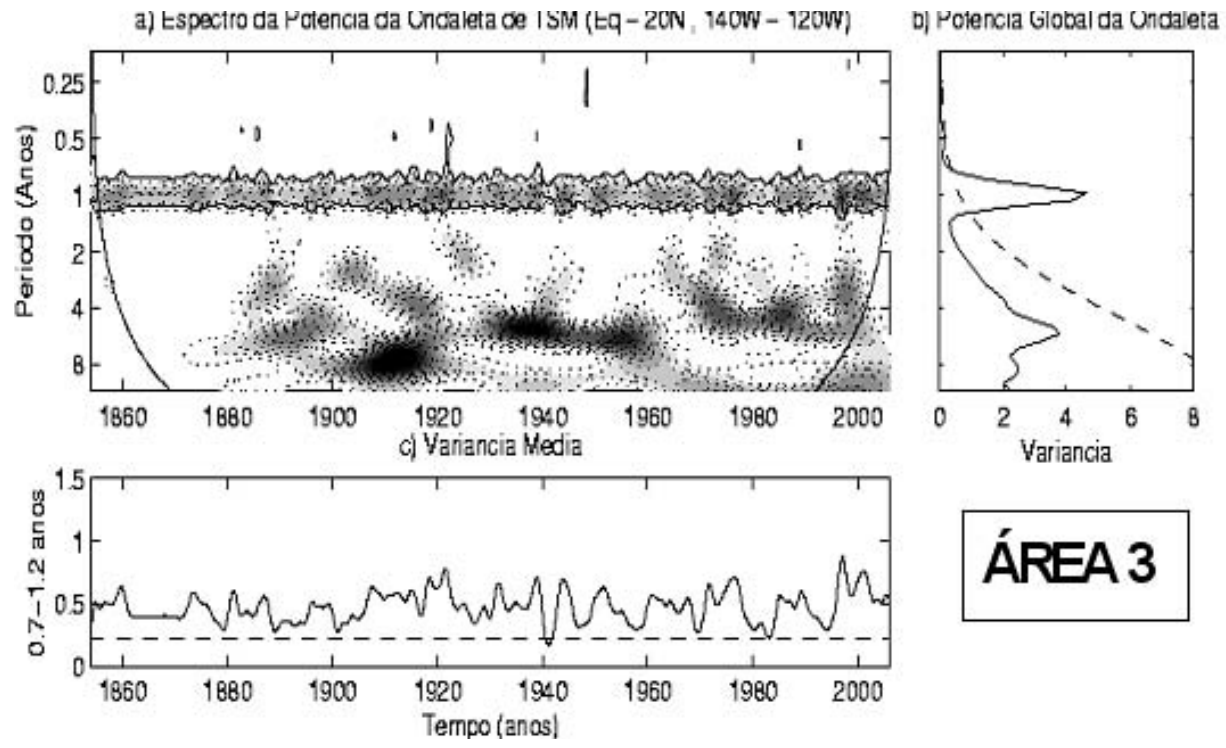

Figura 3 - Idem Figura 2, para a área 3 e $\sigma^{2}=0,4525$. 
áreas 9, 10, 14, 15 e 16 (Figuras 6, 9 e 10 para as áreas 9, 14 e 16 e Figuras não mostradas para as demais áreas), são consideravelmente maiores do que os das áreas do HN. De fato, os gráficos de EGP mostram para a escala anual valores e torno de $10 \sigma$ a $12 \sigma$ para as áreas 9,10 e 14 e de $50 \sigma$ na área 16 . Além disso, a variância na escala anual é crescente da linha da data para leste com um máximo entre $100^{\circ} \mathrm{W}$ e $80^{\circ} \mathrm{W}$ (Figuras 8 a 10). Portanto, a máxima variância anual ocorre em longitudes próximas à costa oeste da América do Sul ao sul do equador. Setoh et al. (1999) também encontraram aumento da variância do ciclo anual de oeste para leste, porém para a faixa entre $2,5^{\circ} \mathrm{N}$ e $2,5^{\circ} \mathrm{S}$. Observa-se também uma pequena variabilidade semianual no EPG na área 9 (Figura 6b). Nas áreas 12 a 16 (Figuras 7 a 9) notam-se picos secundários (não-significativos) de variabilidade interanual (escala de 5 anos), que se reduzem gradualmente para leste.

Similar ao HN, as MEPOs para a escala de 0,7 -1,2 anos (painel (c) das Figuras 6 a 9) e as correspondentes EPO mostram fraca variabilidade anual e praticamente ausência de sinal da variabilidade interanual para o período anterior a 1880. A fraca
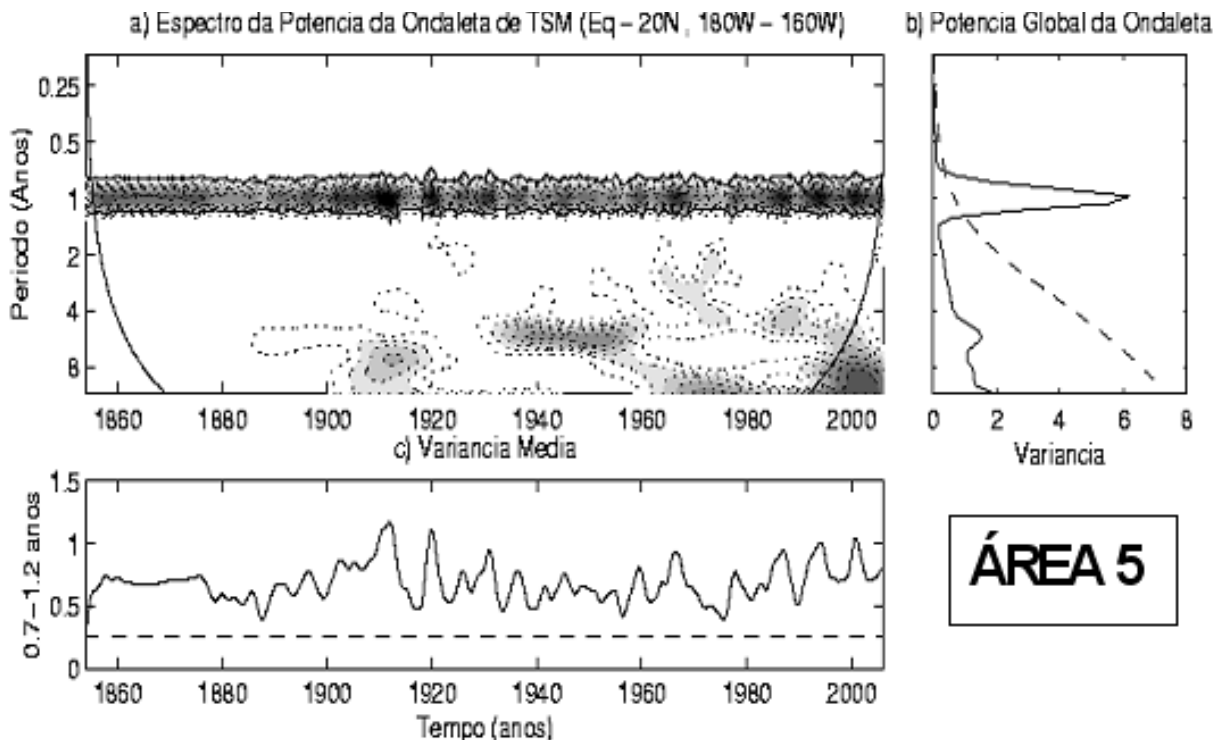

Figura 4 - Idem Figura 2, para a área 5 e $\sigma^{2}=0,4351$.
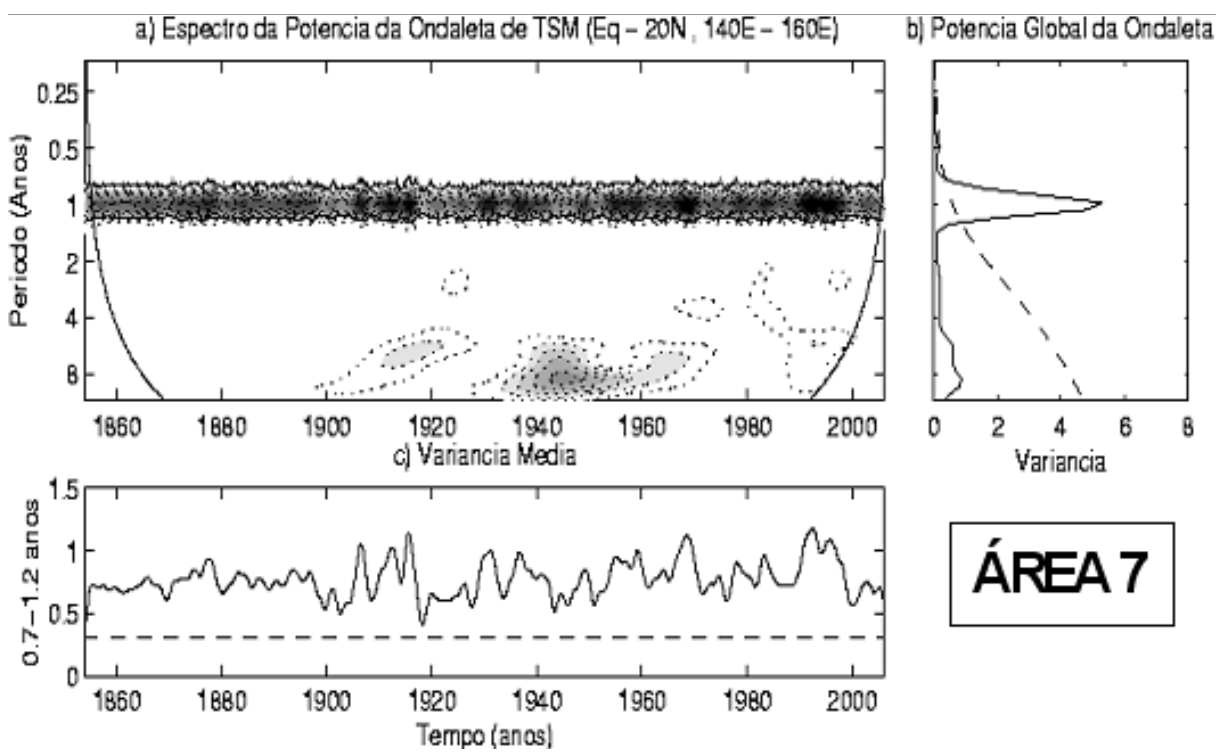

Figura 5 - Idem Figura 2, para a área 7 e $\sigma^{2}=0,3339$. 
variabilidade interanual nos dados de TSM anterior a 1880 foi ressaltada por Smith e Reynolds (2004). A MEPO da área 9 (Figura 6) mostra forte variabilidade interanual na maior parte do período de estudo, com picos de magnitudes similares. Este mesmo comportamento é também notável na MEPO do índice da área 10 (Figura não mostrada), porém com um número maior de picos com valores superiores a $1 \sigma$, como em 1886, 1917, 1919, 1940 e 1982. Destes anos, os dois primeiros coincidem com anos de ocorrências de La Niña e os dois últimos com anos de ocorrências de El Niño. Na MEPO do índice da área 11 (Figura não mostrada) destacam-se um pico de aproximadamente 1,3 se por volta de 1885 e vários picos superiores a $1 \sigma$ em 1900 , 1915, 1930, 1952, 1980, 1986 e 1996, que coincidem com anos de ocorrência de El Niño, exceto em 1996. Assim, os ciclos anuais correspondentes aos índices de TSM nas áreas 9 a 11 têm forte variabilidade interanual, a qual pode estar relacionada à ocorrência de extremos do ENOS, como em 1886 e 1917 (anos de La Niña) e em 1900, 1915, 1930, 1940, 1952, 1980, 1982 e 1986 (anos de ocorrência de El Niño). Os gráficos da MEPO para os índices das áreas 12 (Figura 8c) e 13 (Figura não mostrada) apresentam menor variabilidade, de modo que os valores dos

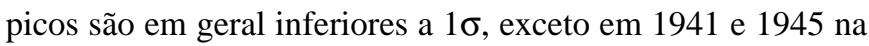
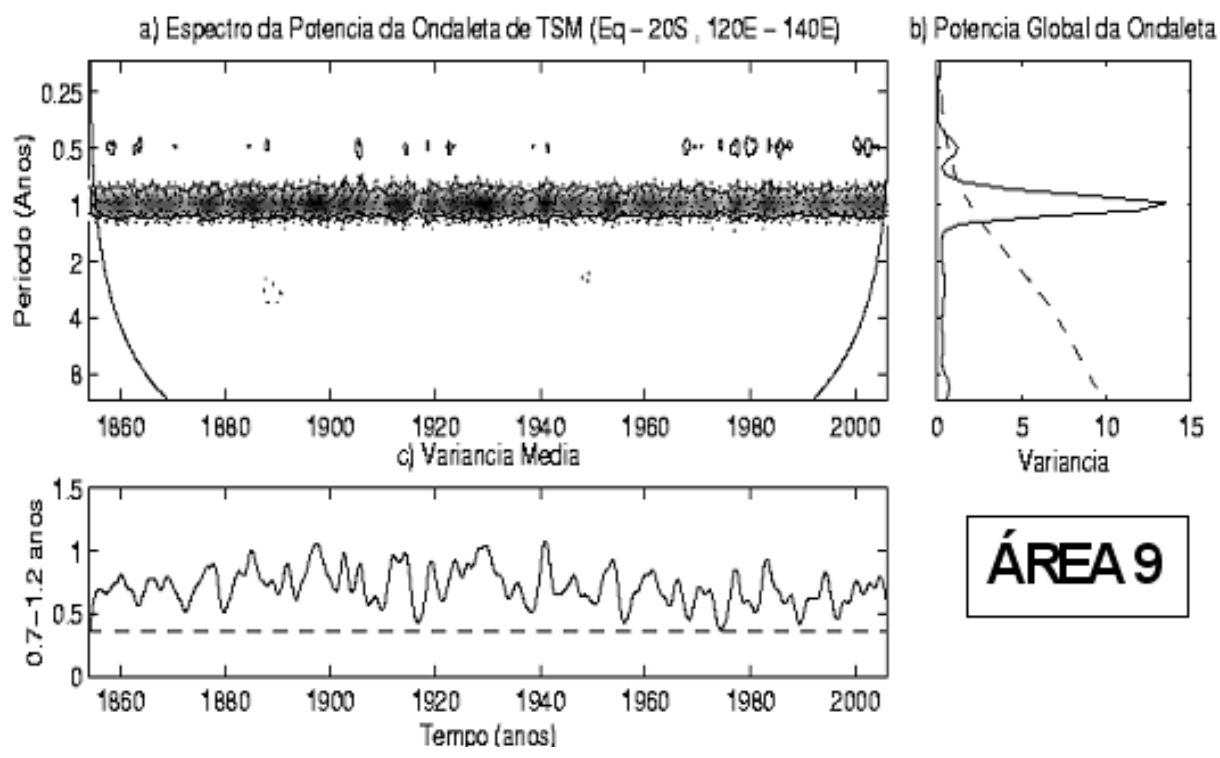

\section{ÁREA 9}

Figura 6 - Idem Figura 2, para a área 9 e $\sigma^{2}=0,9160$.
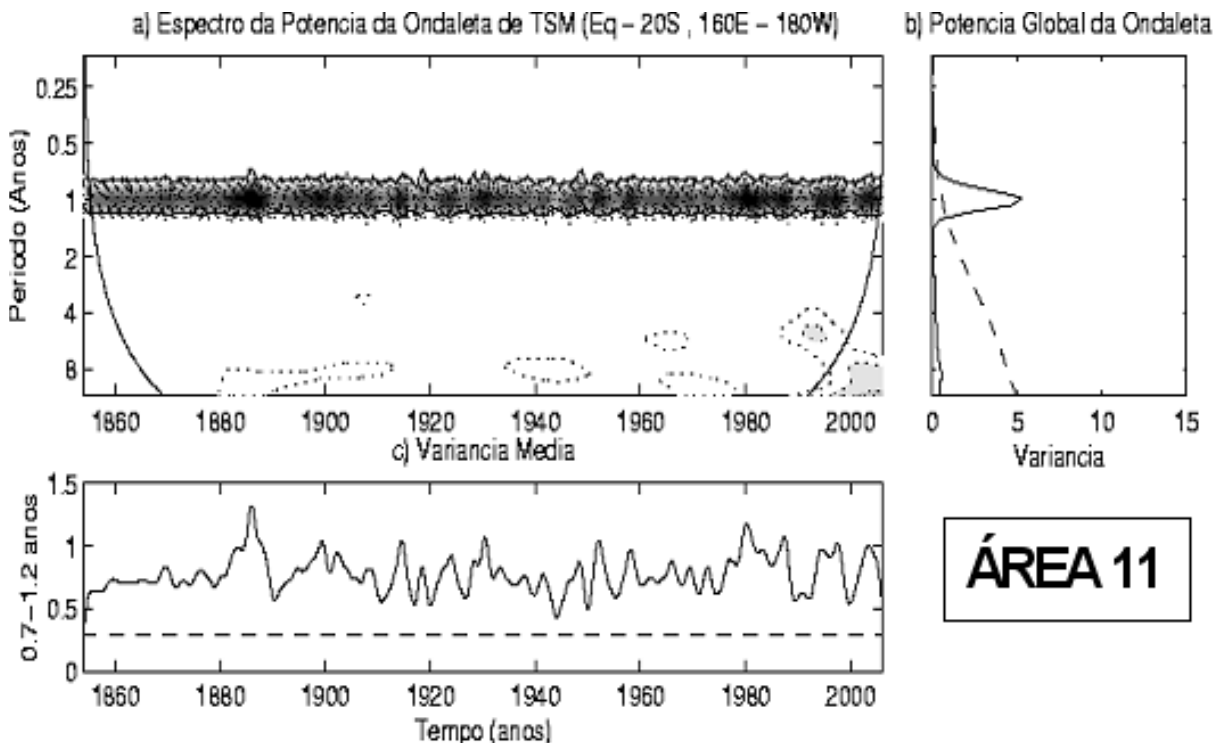

\section{ÁREA 11}

Figura 7 - Idem Figura 2, para a área 11 e $\sigma^{2}=0,3304$. 
área 13, quando picos ligeiramente superiores a $1 \sigma$ são notados. As MEPOs das áreas 14 a 16 mostram maior variabilidade, com picos superiores a $1 \sigma$, destacando-se em particular os picos em 1941 e 1945 nas áreas 14 e 15, e em 1890, 1950 e 2000 na área 16 que coincidem com anos de ocorrência de La Niña (Figuras 9 e 10 para as áreas 14 e 16, e Figura não mostrada para a área 15).

As análises acima mostram diferenças importantes na variabilidade do ciclo anual de TSM no Pacífico tropical entre o HN e HS. No setor norte o valor do EPG aumenta de leste para oeste, e o valor máximo está entre $120^{\circ} \mathrm{E}$ e $140^{\circ} \mathrm{E}$ (área 8), na região da piscina de águas quentes do Pacífico tropical norte oeste. Por outro lado, nas áreas ao sul do equador a variância é crescente da linha da data para leste com um máximo entre $100^{\circ} \mathrm{W}$ e $80^{\circ} \mathrm{W}$. Com relação à variabilidade interanual, esta é maior mais a oeste, entre $120^{\circ} \mathrm{E}$ e $140^{\circ} \mathrm{E}$, para a faixa entre equador e $20^{\circ} \mathrm{N}$ e no Pacífico central para a faixa entre equador e $20^{\circ} \mathrm{S}$, porém nesta escala não ocorrem picos significativos nas correspondentes EPGs.

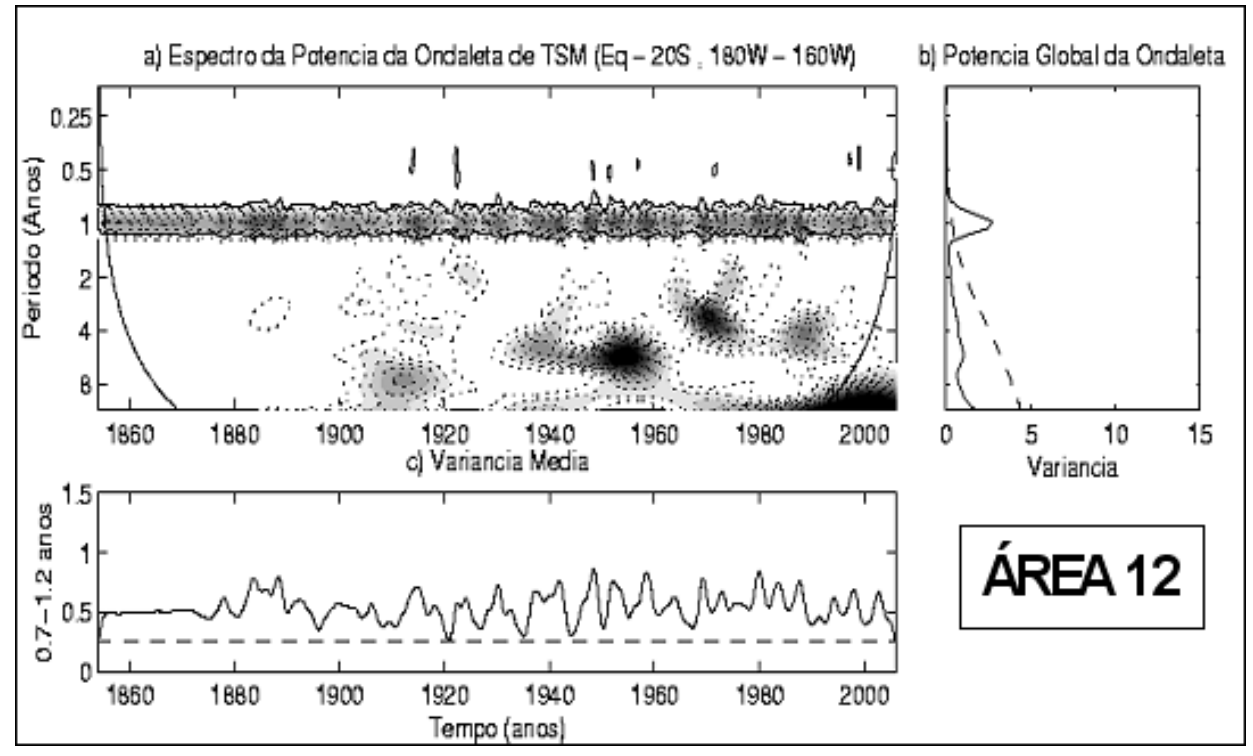

Figura 8 - Idem Figura 2, para a área 12 e $\sigma^{2}=0,2463$.
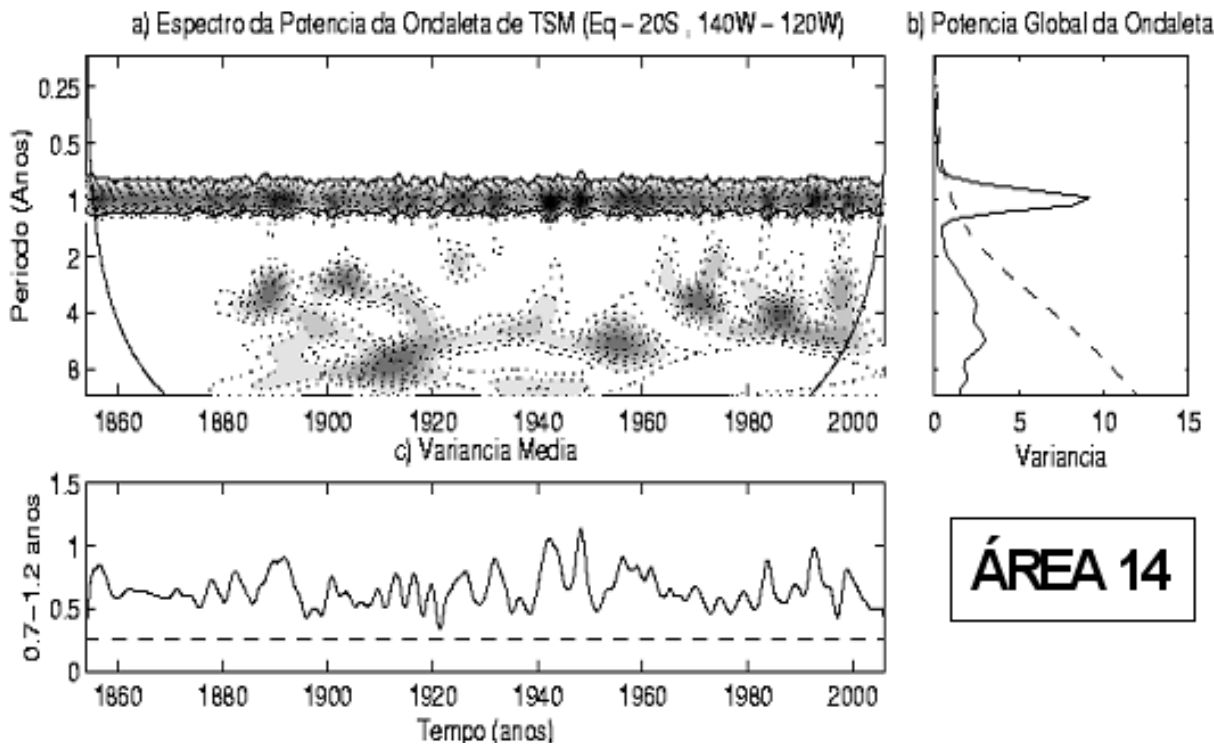

Figura 9 - Idem Figura 2, para a área 14 e $\sigma^{2}=0,6796$. 


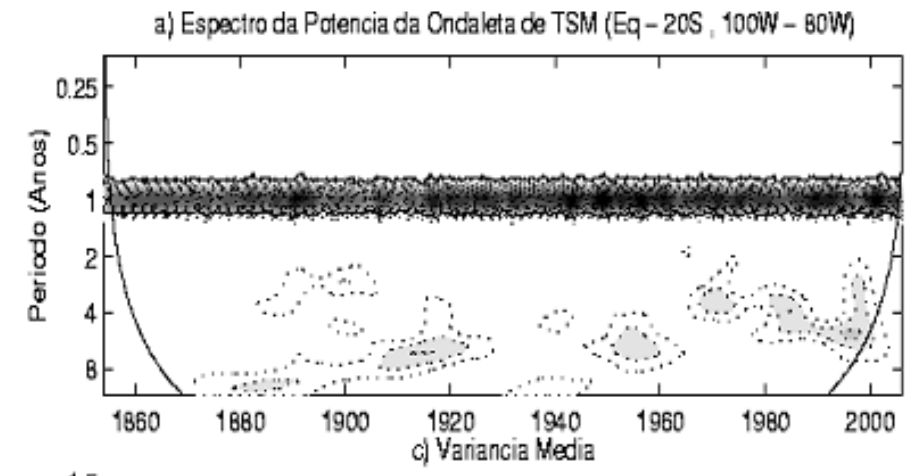

b) Poiencia Global da Ondaleta
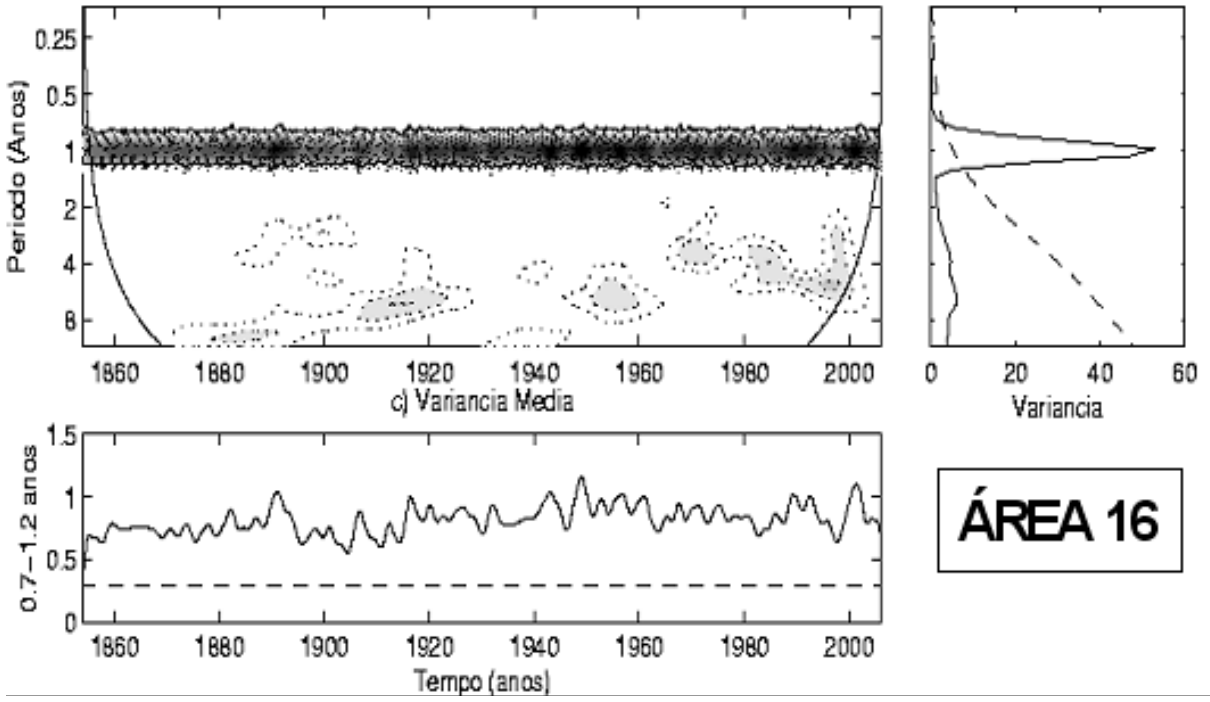

Figura 10 - Idem Figura 2, para a área 16 e $\sigma^{2}=3,1444$.

\section{CONCLUSÕES}

O ciclo anual de TSM no Pacífico tropical entre $20^{\circ} \mathrm{N}$ e $20^{\circ} \mathrm{S}$ foi analisado considerando os dados desta variável disponíveis de 1854 a 2006. As análises foram baseadas em índices de TSM de áreas entre o equador e $20^{\circ} \mathrm{N}$ e equador e $20^{\circ} \mathrm{S}$ com $20^{\circ}$ de extensão em longitude. A TO foi a técnica adotada por permitir uma análise detalhada de variações tempo-freqüência de cada índice. As análises são baseadas nas quantidades derivadas de análises usando TO.

O ciclo anual de TSM possui variabilidade interanual e interdecenal como mostrado nas análises das MEPOs. O principal resultado do presente trabalho é que em grande parte, a variabilidade interanual no ciclo anual de TSM das áreas do HN e HS está relacionada ao ciclo do ENOS. De fato, a maioria dos picos da MEPO para 0,7 a 1,2 anos para os índices do HN e HS, coincide com anos de extremos do ENOS. No caso do HN, ocorreram picos na MEPO nos anos de El Niño de 1976 e 1993 na área 1, de 1888 na área 2, de 1992 na área 5, de 1931 e 1992 na área 6, de 1930, 1958, 1969 e 1992 na área 7 e de 1969 na área 8; e nos anos de La Niña de 1880, 1918, 1942 e 2000 na área 1, de 1942 na área 2, de 1911, 1920, 2000 na área 5, de 1907, 1920 e 1985 na área 6, e de 1908, 1918 e 1938 na área 7. No caso do HS, ocorreram picos na MEPO nos anos de El Niño de 1940 e 1982 na área 10, de 1885, 1900, 1915, 1930, 1952, 1980 e 1986 na área 11 e nos anos de La Niña de 1886, 1917 na área 10 e de 1890, 1950 e 2000 na área 16. Vale, no entanto, salientar que alguns picos na MEPO para 0,7 a 1,2 anos das áreas tanto do HN como do HS, não coincidem com anos de extremos do ENOS. Nestes casos os picos na MEPO se devem a outros fatores não relacionados ao ENOS.

As análises dos EPGs mostram que a variância do ciclo anual de TSM para o HN cresce gradualmente de leste para oeste, e atinge um valor máximo entre $120^{\circ} \mathrm{E}$ e $140^{\circ} \mathrm{E}$ na região da piscina de águas quentes do Pacífico tropical oeste e norte . Por outro lado, para o HS tal variância é crescente da linha da data para leste com o máximo entre $100^{\circ} \mathrm{W}$ e $80^{\circ} \mathrm{W}$. Setoh et al. (1999) também encontraram aumento da amplitude do ciclo anual de TSM da linha da data para leste, porém considerando apenas a faixa entre $2,5^{\circ} \mathrm{N}$ e $2,5^{\circ} \mathrm{S}$. Assim, os resultados de Setoh et al. (1999) se estendem para latitudes mais ao sul do que somente para a faixa equatorial. Os resultados do presente trabalho mostram que a máxima variância do ciclo anual de TSM apresenta variações distintas de um hemisfério para o outro.

Os resultados aqui apresentados sugerem, que estudos futuros sobre as interações entre as variabilidades anual e interanual de TSM no Pacífico, devem levar em conta as diferenças no ciclo anual de TSM entre os hemisférios norte e sul.

\section{AGRADECIMENTOS}

Este artigo foi derivado do trabalho de dissertação de mestrado do primeiro autor. Os autores agradecem à Coordenação de Aperfeiçoamento de Pessoal de Nível Superior (CAPES) pela bolsa de Mestrado cedida por dois anos ao primeiro autor deste trabalho. Os autores agradecem aos dois revisores pelos comentários apresentados, os quais contribuíram substancialmente para melhorar o artigo. 


\section{REFERÊNCIAS BIBLIOGRÁFICAS}

ACEITUNO, P. On the functioning of the Southern Oscillation in the South American sector. Part 1: surface climate. Monthly Weather Review, v. 116, n. 3, p. 505-524, 1988.

BJERKNES, J. Atmospheric teleconnections from the equatorial Pacific. Monthly Weather Review, v.97,n. 3, p.163-172, 1969.

GRIMM, A. M.; BARROS, V. R.; DOYLE, M. E. Climate variability in southern South America associated with El Nino and La Nina events. Journal of Climate, v. 13, n. 1, p. 35-58, 2000.

GRIMM, A. M.; FERRAZ, S. E.; GOMES, J. Precipitation anomalies in southern Brazil associated with El Nino and La Nina events. Journal of Climate, v. 11, n. 11, p. 2863-2880, 1998.

GU, D.; PHILANDER, G. H. Secular changes of annual and interannual variability in the tropics during de past century. Journal of Climate, v.8, n. 4, p.864-876, 1995.

KAYANO, M. T.; RAO, V. B.; ANDREOLI, R. V. A review of short-term climate variability mechanisms. Advances in Space Research, v. 35, n. 5, p.843-851, 2005.

KAYANO, M. T.; RAO, V. B.; MOURA, A. D. Tropical circulations and the associated rainfall anomalies during two contrasting years. Journal of Climatology, v. 8, n. 5, p. 477-488, 1988.

KIM, K.Y.; CHUNG, C. On the evolution of the annual cycle in tropical Pacific. Journal of Climate, v. 14, n. 5, p.991-994, 2001.

KOUSKY, V. E.; ROPELEWSKI, C. F. Extremes in the Southern Oscillation and their relationship to precipitation anomalies with emphasis on the South American region. Revista Brasileira de Meteorologia, v. 4, n. 1, p. 351-363, 1989.

RAO, V. B.; HADA, K. Characteristics of rainfall over Brazil: annual and variations and connections with the Southern Oscillation. Theoretical and Applied Climatology, v. 42, p. 81-91, 1990.

RASMUSSON, E. M.; ARKIN, P. A. Interannual climate variability associated with the El Niño/Southern
Oscillation. Amsterdam: Elsevier Science Publishers B. V., chapter 40, p. 697-725, 1985.

RASMUSSON, E. M.; CARPENTER, T. H. Variations in tropical sea surface temperature and surface wind fields associated with the Southern Oscillation/El Niño. Monthly Weather Review, v. 110, n. 5, p.354-384, 1982.

ROPELEWSKI, C. F.; HALPERT, M. S. Global and regional scale precipitation patterns associated with the El Niño/Southern Oscillation. Monthly Weather Review, v. 115, n. 8, p.1606-1626, 1987.

ROPELEWSKI, C. F.; HALPERT, M. S. Precipitation patterns associated with the high index phase of the Southern Oscillation. Journal of Climate, v. 2, n. 3, p. 268-284, 1989.

ROPELEWSKI, C. F; HALPERT, M. S. Quantifying Southern Oscillation-precipitation relationships. Journal of Climate, v. 9, n. 5, p.1043-1059, 1996.

SETOH, T.; IMAWAKI, S.; OSTROVSKII, A.; UMATANI, S. Interdecadal variations of ENSO signals and annual cycles revealed by wavelet analysis. Journal of Oceanography, v. 55, n. 3, p.385- 394, 1999.

SMITH, T. M.; REYNOLDS, R. W. Improved extended reconstruction of SST (1854-1997). Journal of Climate, v. 17, n. 12, p. 2466-2477, 2004.

TORRENCE, C.; COMPO, G. P. A practical guide to wavelet analysis. Bulletin of the American Meteorological Society, v. 79, n. 1, p.61-78, 1998.

TRENBERTH, K. E. The definition of El Niño. Bulletin of the American Meteorological Society, v. 78, n. 12, p. 2771-2777, 1997.

WANG, B. Interdecadal changes in El Niño onset in the last four decades. Journal of Climate, v. 8, n. 2, p. 267-285, 1995.

WANG, B.; WANG Y. Temporal structure of the Southern Oscillation as revealed by waveform and wavelet analysis. Journal of Climate, v. 9, n. 7, p. 1586-1598, 1996.

XUE, Y.; SMITH, T. M.; REYNOLDS, R. W. Interdecadal changes of 30-Yr SST normals during 1871-2000. Journal of Climate, v. 16, n. 10, p. 1601-1612, 2003. 